Pacific Journal of Mathematics

ON ANTI-COMMUTATIVE ALGEBRAS AND GENERAL LII 


\title{
ON ANTI-COMMUTATIVE ALGEBRAS AND GENERAL LIE TRIPLE SYSTEMS
}

\author{
Arthur A. SAgLe
}

A general Lie triple system as defined by $K$. Yamaguti, is considered as an anti-commutative algebra $A$ with a trilinear operation $[x, y, z]$ in which (among other things) the mappings $D(x, y): z \rightarrow[x, y z]$ are derivations of $A$. It is shown that if the trilinear operation is homogeneous, and $A$ is irreducible as a general L. t. s. or irreducible relative to the Lie algebra $I(A)$ generated by the $D(x, y)$ 's, then $A$ is a simple algebra. The main result is the following. If $A$ is a simple finitedimensional anti-commutative algebra over a field of characteristic zero which is a general L.t. $\mathrm{s}$. with a homogeneous trilinear operation $[x, y, z]$, then $A$ is (1) a Lie algebra; or (2) a Malcev algebra; or (3) an algebra satisfying $J(x, y, z) w=$ $J(w, x, y z)+J(w, y, z x)+J(w, z, x y)$ where $J(x, y, z)=x y \cdot z+$ $y z \cdot x+z x \cdot y$. Furthermore in all three cases $I(\mathbf{A})$ is the derivation algebra of $A$ and $I(A)$ is completely reducible in $A$.

1. A general Lie triple system (general L. t. s.) has been defined in [6] to be a vector space $V$ over a field $F$ which is closed with respect to_a trilinear operation $[x, y, z]$ and a bilinear operation $x y$ so that

$$
\begin{gathered}
{[x, y, z]=0} \\
x^{2}=0 \\
{[x, y, z]+[y, z, x]+[z, x, y]-(x y) z-(y z) x-(z x) y=0,} \\
{[w x, y, z]+[x y, w, z]+[y w, x, z]=0,} \\
{[[u, v, w], x, y]+[[v, u, x], w, y]} \\
+[v, u,[w, x, y]]+[w, x,[u, v, y]]=0, \\
{[w, x, y z]+z[w, x, y]+y[x, w, z]=0 .}
\end{gathered}
$$

A general L.t.s. is an extension of a Lie triple system used in differential geometry and Jordan algebras. Next we note that if $V$ is a Lie algebra with multiplication $x y$, then $V$ becomes a general L.t.s. by setting $[x, y, z]=(x y) z$. As an extension of this it was shown in [7] that if $V$ is a Malcev algebra [2] with multiplication $x y$, then $V$ becomes a general L. t. s. by setting $[x, y, z]=-(x y) z+(y z) x+(z x) y$.

Received January 23, 1964. Sponsored by the U.S. Army Research Office (Durham) and by NSF Grant GP-1453. 
In this paper we shall take the point of view that a general L.t.s. is an algebra $A$ with multiplication $x y$ satisfying (1.1) - (1.6); that is, $A$ is an anti-commutative algebra with linear transformations

$$
D(x, y): A \rightarrow A: z \rightarrow z D(x, y) \equiv[x, y, z]
$$

for all $x, y \in A$ satisfying (1.1)-(1.6). Thus from (1.6) we see that each $D(x, y)$ is a derivation of $A$ satisfying various identities. Motivated by the above examples we shall assume that the trilinear product $[x, y, z]$ is a linear homogeneous expression in the products of $x, y$ and $z$. Thus using anti-commutativity we assume there exist fixed $\alpha$, $\beta, \gamma \in F$ so that

(1.7) $[x, y, z]=\alpha x y \cdot z+\beta y z \cdot x+\gamma z x \cdot y$.

With (1.7) as the form for the trilinear operation, we next consider irreducibility conditions on $A$. First suppose $A$ is irreducible as a general L.t.s.; that is, $A$ has no proper general L.t.s. subspace $B$ so that $[B, A, A] \subset B$, then $A$ is a simple algebra. For if $B$ is a proper ideal of $A$, then from (1.7) $B$ is general L.t. s. subspace so that $[B, A, A] \subset B$. Next let $I(A)$ be the subspace of the derivation Lie algebra $D(A)$ generated by all derivations of the form $D(x, y)$, then from (1.5) $I(A)$ is a Lie subalgebra of $D(A)$ under commutation. If $A$ is $I(A)$-irreducible, then by (1.7) $A$ is a simple algebra. Motivated by these remarks the main result is the following

THEOREM. If $A$ is a simple finite dimensional anti-commutative algebra over a field of characteristic zero with a nonzero trilinear operation $[x, y, z]$ satisfying (1.1)-(1.7), then

(1) $A$ is a simple Lie algebra with $\beta=\gamma, \alpha-\beta=1$; or

(2) $A$ is a simple Malcev algebra [4] with $\alpha=-1, \beta=\gamma=1$; or

(3) $A$ is a simple algebra satisfying $J(x, y, z) w=J(w, x, y z)+$ $J(w, y, z x)+J(w, z, x y)$ with $\alpha=1 / 2, \beta=\gamma=1 / 4$ and $J(x, y, z)=$ $x y \cdot z+y z \cdot x+z x \cdot y$.

Furthermore in all three cases $I(A)$ is the derivation algebra of $A$ and $I(A)$ is completely reducible in $A$.

It should be noted that since the trilinear operation $[x, y, z]$ given by (1.7) is homogeneous, any nonzero scalar multiple would also be an admissible trilinear operation. Therefore all superfluous nonzero scalars will be eliminated to obtain the final above normalizations for $\alpha, \beta$ and $\gamma$.

2. Identities. We investigate the identities (1.1)-(1.7) with the assumption that $A$ is a simple finite dimensional anti-commutative 
algebra over a field $F$ of characteristic zero and with multiplication denoted by $x y$. From (1.1),

$$
\begin{aligned}
0=[x, x, y] & =\alpha x x \cdot y+\beta x y \cdot x+\gamma y x \cdot x \\
& =(\beta-\gamma) x y \cdot x .
\end{aligned}
$$

Thus $\gamma=\beta$ or $x y \cdot x=0$ for all $x, y \in A$. Suppose this last equation holds then we must have

$$
x y \cdot z+x \cdot y z=0 \quad x, y, z \in A .
$$

Now let $0 \neq b \in A$ and consider $B=b A . \quad B$ is an ideal of $A$; for if $y, z \in A$, then $b y \cdot z=-b \cdot y z$. Thus $B=0$ or $B=A$. $B=0$ implies $b F$ is an ideal of $A$ and therefore $A=b F$. This implies $A^{2}=0$, a contradiction to the simplicity of $A$. But if $R_{x}$ or $R(x)$ denotes the mapping $a \rightarrow a x$, then $B=\mathrm{A}$ implies $R_{b}$ is surjective and since $A$ is finite dimensional, $R_{b}$ is injective. This contradicts $b R_{b}=0$ with $b \neq 0$. Thus we must have $\gamma=\beta$.

From (1.2), $x y=-y x$ which is just the statement that $A$ is anti-commutative.

From (1.3), $\gamma=\beta$ and setting $J(x, y, z)=x y \cdot z+y z \cdot x+z x \cdot y$ we have

$$
\begin{aligned}
J(x, y, z) \doteq & \alpha(x y \cdot z+y z \cdot x+z x \cdot y) \\
& +\beta(y z \cdot x+z x \cdot y+x y \cdot z) \\
& +\gamma(z x \cdot y+x y \cdot z+y z \cdot x) \\
= & (\alpha+2 \beta) J(x, y, z) .
\end{aligned}
$$

Thus if $A$ is not a Lie algebra we have

$$
1=\alpha+2 \beta \text {. }
$$

In case $A$ is a Lie algebra, then $\beta=\gamma$ and $D(x, y)=(\beta-\alpha) R(x y)$. The remaining identities give no more information and therefore the first part of the main theorem is proved by setting $\beta-\alpha=-1$. Henceforth $A$ is assumed to be a non-Lie algebra.

From (1.4) we obtain

$$
\begin{aligned}
0= & \alpha[(w x \cdot y) z+(x y \cdot w) z+(y w \cdot x) z] \\
& +\beta[y z \cdot w x+w z \cdot x y+x z \cdot y w \\
& +(z \cdot w x) y+(z \cdot x y) w+(z \cdot y w) x] \\
= & \alpha J(w, x, y) z \\
& +\beta[y z \cdot w x+(z \cdot w x) y+(w x \cdot y) z-(w x \cdot y) z \\
& +w z \cdot x y+(z \cdot x y) w+(x y \cdot w) z-(x y \cdot w) z \\
& +x z \cdot y w+(z \cdot y w) x+(y w \cdot x) z-(y w \cdot x) z]
\end{aligned}
$$




$$
\begin{aligned}
= & \alpha J(w, x, y) z-\beta J(w, x, y) z \\
& +\beta[J(y, z, w x)+J(w, z, x y)+J(x, z, y w)] .
\end{aligned}
$$

Thus

$$
(\alpha-\beta) J(w, x, y) z=\beta[J(z, w, x y)+J(z, x, y w)+J(z, y, w x)] .
$$

From (2.2) we see that $\beta \neq 0$. For suppose $\beta=0$, then from (2.1), $\alpha=1$ and from (2.2), $J(w, x, y) z=0$. Now if $J(A, A, A)$ denotes the subspace spanned by the elements $J(w, x, y)$ for all $w, x, y \in A$ we see that $J(A, A, A)$ is a nonzero ideal of $A$ and so $A=J(A, A, A)$. But $J(w, x, y) z=0$ implies $A^{2}=0$, a contradiction.

We rewrite (1.5) in terms of the derivations $D(u, v)$ by operating on $y$ in (1.5) to obtain

$$
[D(w, x), D(v, u)]=D(w D(v, u), x)+D(w, x D(v, u)),
$$

where for linear transformations $S$ and $T,[S, T]=S T-T S$. We shall not use this identity since a straightforward computation, as suggested by the referee, shows (1.6) and (1.7) imply (1.5).

Next using $[x, y, z]=(\alpha-\beta) x y \cdot z+\beta J(x, y, z)$ we obtain from (1.6),

$$
\begin{aligned}
0= & (\alpha-\beta) w x \cdot y z+\beta J(w, x, y z) \\
& +(\alpha-\beta) z(w x \cdot y)+\beta z J(w, x, y) \\
& +(\alpha-\beta) y(x w \cdot z)+\beta y J(x, w, z)
\end{aligned}
$$

and therefore

$$
\begin{aligned}
& (\alpha-\beta) J(w x, y, z)-\beta J(y z, w, x) \\
& \quad=\beta[J(w, x, z) y-J(w, x, y) z]
\end{aligned}
$$

3. Proof of main theorem. We shall investigate first the restrictions imposed by (2.2) and (2.4). In (2.4) set $w=y$ and $z=x$ to obtain

$$
\begin{aligned}
(\alpha-2 \beta) J(x y, x, y) & =\beta[J(y, x, x) y-J(y, x, y) x] \\
& =0 .
\end{aligned}
$$

Thus we have

CASE I.

$$
J(x y, x, y)=0, \quad \text { or }
$$

CASE II.

$$
\alpha=2 \beta \text {. }
$$

We shall show that in Case I, A must be a non-Lie Malcev algebra (since we are assuming $A$ is not a Lie algebra) and that Case II yields an anti-commutative algebra satisfying $J(x, y, z) w=J(w, x, y z)+$ $J(w, y, z x)+J(w, z, x y)$. 
For Case I we linearize $J(x y, x, y)=0$ to obtain

$$
\begin{aligned}
& J(w x, y, z)+J(y z, w, x)=J(w y, z, x)+J(z x, w, y), \\
& w J(x, y, z)-x J(y, z, w)+y J(z, w, x)-z J(w, x, y) \\
& \quad=3[J(w x, y, z)+J(y z, w, x)] .
\end{aligned}
$$

Using (3.2) and (2.2) we have

$$
\begin{aligned}
& 3(\alpha-\beta)[J(w x, y, z)+J(y z, w, x)] \\
= & (\alpha-\beta)[-J(x, y, z) w+J(y, z, w) x-J(z, w, x) y+J(w, x, y) z] \\
= & \beta[-J(w, x, y z)-J(w, y, z x)-J(w, z, x y) \\
& +J(x, y, z w)+J(x, z, w y)+J(x, w, y z) \\
& -J(y, z, w x)-J(y, w, x z)-J(y, x, z w) \\
& +J(z, w, x y)+J(z, x, y w)+J(z, y, w x)] \\
= & \beta[2 J(x y, z, w)+2 J(z w, x, y) \\
& -2 J(w x, y, z)-2 J(y z, w, x) \\
& +2 J(w y, x, z)+2 J(x z, w, y)] \\
= & -6 \beta[J(w x, y, z)+J(y z, w, x)], \quad \text { using (3.1). }
\end{aligned}
$$

Thus

$$
\begin{gathered}
\alpha-\beta=-2 \beta \neq 0 \quad \text { or } \\
J(w x, y, z)+J(y z, w, x)=0 .
\end{gathered}
$$

Now in case $\alpha-\beta=-2 \beta$ we have from (2.2),

$$
2 z J(w, x, y)=J(z, w, x y)+J(z, x, y w)+J(z, y, w x)
$$

and using this identity with (3.1) we have

$$
\begin{aligned}
2 w J(w, x, y) & =J(w, x, y w)+J(w, y, w x) \\
& =2 J(w, x, y w) .
\end{aligned}
$$

Thus $A$ is a Malcev algebra. Using the results of [3] we may assume $\alpha=-1, \beta=\gamma=1$. Also from [3] the derivation algebra equals $I(A)$ and $I(A)$ is completely reducible in $A$.

So we next assume $A$ satisfies (3.3), then using (2.4) - we obtain

$$
\alpha J(w x, y, z)=\beta[J(w, x, z) y-J(w, x, y) z],
$$

and therefore

$$
\begin{aligned}
\alpha(\alpha-\beta) J(w x, y, z)= & \beta(\alpha-\beta)[J(w, x, z) y-J(w, x, y) z] \\
= & \beta^{2}[J(y, w, x z)+J(y, x, z w) \\
& +J(y, z, w x)-J(z, w, x y) \\
& -J(z, x, y w)-J(z, y, w x)], \quad \text { using } \\
= & 2 \beta^{2} J(w x, y, z), \quad \text { using (3.3). }
\end{aligned}
$$


Thus

$$
\begin{array}{ll}
J(w x, y, z)=0, & \text { or } \\
\alpha(\alpha-\beta)=2 \beta^{2} . &
\end{array}
$$

In the first case, $A$ is a Lie algebra which is a contradiction and in the second case $\alpha=-\beta$ or $2 \beta$. Thus as subcases we have

Case A. $\quad \alpha=-\beta$ and therefore $\alpha=-1, \beta=\gamma=1$;

Case B. $\quad \alpha=2 \beta$ and therefore $\alpha=1 / 2, B=\gamma=1 / 4$.

First consider Case A, then from (2.2) and (3.3) we have

$$
\begin{aligned}
2 w J(w, x, y) & =J(w, w, x y)+J(w, x, y w)+J(w, y, w x) \\
& =2 J(w, x, y w)
\end{aligned}
$$

and therefore $A$ is a Malcev algebra. We shall next show that this Malcev algebra of Case A actually does not exist. First for any anti-commutative algebra $A$ define the linear transformation $\Delta(x, y)$ by

$$
z \Delta(x, y)=J(x, y, z)
$$

and let $\Delta(A, A)$ be the linear space of transformations spanned by these $A(x, y)$ 's for all $x, y \in A$. Using (3.3) we have

$$
\begin{aligned}
0 & =J(w x, y, z)+J(w, x, y z) \\
& =w\left(R_{x} \Delta(y, z)+\Delta(x, y z)\right)
\end{aligned}
$$

and therefore

$$
R_{x} \Delta(y, z)=-\Delta(x, y z) \in \Delta(A, A) .
$$

From identities (2.32) and (2.34) of [2] we also have

$$
2 \Delta(y, z) R_{x}=2 R_{x} \Delta(y, z)-2 R(J(x, y, z))-4 \Delta(y z, x) \in \Delta(A, A),
$$

using also the preceding identity. Thus we see from these identities that $A(A, A)$ is an ideal in the transformation algebra $T(A)$ which is generated by $R(A)=\left\{R_{x}: x \in A\right\}$. But since $A$ is simple, $T(A)$ is a simple associative algebra and therefore $\Delta(A, A)=0$ or $T(A)=\Delta(A, A)$. But $\Delta(A, A)=0$ implies $A$ is a Lie algebra and therefore $\Delta(A, A)$ is a simple associative algebra. But we shall next show that $A(x, y)^{2}=$ 0 and therefore conclude that $\Delta(A, A)$ have a basis consisting of nilpotent elements. Thus $\Delta(A, A)$ must be a nilpotent associative algebra, a contradiction to the simplicity of $\Delta(A, A)$. Hence Case A does not exist. So to show $\Delta(x, y)^{2}=0$ we have

$$
\begin{aligned}
-2 z \Delta(x, y) R(x y)= & 2 x y \cdot J(x, y, z) \\
= & J(x y, x, y z)+J(x y, y, z x) \\
& +J(x y, z, x y)
\end{aligned}
$$




$$
\begin{aligned}
= & -J(x \cdot y z, x, y)-J(y \cdot z x, x, y) \\
& -J(z \cdot x y, x, y), \quad \text { using }(3.3) \\
= & J(J(x, y, z), x, y) \\
= & z \Delta(x, y)^{2},
\end{aligned}
$$

that is, $\Delta(x, y)^{2}=-2 \Delta(x, y) R(x y)$. But from identity (2.33) of [2], $\Delta(x, y)^{2}=-3 \Delta(x, y) R(x y)$ and therefore $\Delta(x, y)^{2}=0$.

Next we derive more identities for Case B and use methods similar to those used in Case A to show Case B does not exist either. Using the notation from Case A we obtain again from (3.3) the identity (3.4). Also with $\alpha=1 / 2, \beta=1 / 4$ in (2.2) we obtain

$$
J(w, x, y) z=J(z, w, x y)+J(z, x, y w)+J(z, y, w x) .
$$

From (3.5) and (3.4) we have

$$
\Delta(x, y) R_{z}=-\Delta(z, x y)-R_{y} \Delta(z, x)+R_{x} \Delta(z, y) \in \Delta(A, A) .
$$

Thus as in Case $\mathrm{A}$ we see $\Delta(A, A)=T(A)$ is a simple associative algebra. Next from (3.5) we also have

$$
R(J(w, x, y))=\Delta(w x, y)+\Delta(x y, w)+\Delta(y w, x),
$$

and using (3.4) we obtain

$$
\begin{aligned}
\Delta(x, y)^{2} & =\left[R_{x}, R_{y}\right] \Delta(x, y)-R(x y) \Delta(x, y) \\
& =R_{x} R_{y} \Delta(x, y)-R_{y} R_{x} \Delta(x, y)+\Delta(x y, x y) \\
& =\Delta(x, y \cdot x y)-\Delta(y, x \cdot x y)+\Delta(x y, x y) \\
& =-\Delta(x y \cdot x, y)-\Delta(x y, x y)-\Delta(y \cdot x y, x) \\
& =-R(J(x y, x, y)) \\
& =0,
\end{aligned}
$$

where the last equality follows from (3.3). Thus as in Case A, we conclude that Case $\mathrm{B}$ does not exist, this completes Case I.

Next consider Case II where $\alpha=2 \beta \neq 0$. From (2.2) and (2.4) we see that $A$ satisfies (3.5) and

$$
\begin{aligned}
& J(w x, y, z)-J(y z, w, x) \\
= & J(w, x, z) y-J(w, x, y) z .
\end{aligned}
$$

Next we rewrite (3.5) and (3.7) in terms of right multiplications to obtain (3.6) and

$$
\Delta(z, w x)-R_{z} \Delta(w, x)=-R(J(w, x, z))-\Delta(w, x) R_{z},
$$

by operation on $y$ in (3.7). Using this and (3.6) we have

$$
\Delta(w, x) R_{z}-R_{z} \Delta(w, x)=-\Delta(w x, z)-\Delta(x z, w)
$$




$$
\begin{aligned}
& -\Delta(z w, x)-\Delta(z, w x) \\
= & \Delta(z x, w)-\Delta(z w, x) ;
\end{aligned}
$$

$$
\left[\Delta(w, x), R_{z}\right]=\Delta(z x, w)-\Delta(z w, x) .
$$

Now using (3.8) and the Jacobi identity we have

$$
\begin{aligned}
{[\Delta(w, x), \Delta(u, v)]=} & {\left[\Delta(w, x),\left[R_{u}, R_{v}\right]\right]-[\Delta(w, x), R(u v)] } \\
= & -\left[\left[R_{u}, \Delta(w, x)\right], R_{v}\right]-\left[R_{u},\left[R_{v}, \Delta(w, x)\right]\right. \\
& -[\Delta(w, x), R(u v)] \in \Delta(A, A) .
\end{aligned}
$$

Thus defining the Lie transformation algebra of $A$, denoted by $L(A)$, to be the Lie algebra generated by $R(A)=\left\{R_{x}: x \in A\right\}$ [5], the above calculations prove

Lemma 3.9. $\Delta(A, A)$ is a Lie algebra and $L(A)=\Delta(A, A)$.

For clearly $\Delta(A, A) \subset L(A)=\Sigma_{i} M_{i}$ where $M_{1}=R(A)$ and $M_{i}=$ $\left[M_{i-1}, M_{1}\right]$ for $i>1$. But also since $A=J(A, A, A)$ we have from (3.6) that $M_{1} \subset \Delta(A, A)$ and since $\Delta(A, A)$ is a Lie algebra, $M_{i} \subset \Delta(A, A)$; thus $L(A) \subset \Delta(A, A)$.

Next we consider the center $C$ of $L(A)=\Delta(A, A)$. Since $A$ is simple, $\Delta(A, A)$ is an irreducible Lie algebra over a field of characteristic zero and therefore from [1, Th. 1], $\Delta(A, A)=C \oplus A^{\prime}$ where $\Delta^{\prime}$ is a semi-simple Lie algebra and $C=\{\Delta \in \Delta(A, A):[\Delta, T]=0$ for all $T \in \Delta(A, A)\}$.

Lemma 3.10. $C=0$ and therefore $A(A, A)$ is a semi-simple Lie. algebra.

Proof. Let $\Delta=\Sigma_{i} \Delta\left(x_{i}, y_{i}\right) \in C$, then for any $u, v \in A$ we have

$$
\begin{aligned}
(u v) \Delta & =-v R_{u} \Delta \\
& =-v \Delta R_{u}, \text { since } R_{u} \in \Delta(A, A) \\
& =-v \Delta \cdot u \\
& =u \cdot v \Delta .
\end{aligned}
$$

But next we have

$$
\begin{aligned}
(v u) \Delta & =\Sigma_{i} v u \Delta\left(x_{i}, y_{i}\right) \\
& =\Sigma_{i} J\left(v u, x_{i}, y_{i}\right) \\
& =\Sigma_{i}\left[J\left(x_{i} y_{i}, v, u\right)-J\left(x_{i}, y_{i}, u\right) v+J\left(x_{i}, y_{i}, v\right) u\right], \\
& \quad J(z, v, u)-\Sigma_{i} u \Delta\left(x_{i}, y_{i}\right) v+\Sigma_{i} v \Delta\left(x_{i}, y_{i}\right) u, \\
& \quad \text { where } z=\Sigma_{i} x_{i} y_{i}
\end{aligned}
$$




$$
\begin{aligned}
& =J(z, v, u)-u \Delta \cdot v+v \Delta \cdot u \\
& =J(z, v, u)+v \cdot u \Delta-u \cdot v \Delta \\
& =J(z, v, u)+(v u) \Delta-(u v) \Delta .
\end{aligned}
$$

Therefore $(u v) \Delta=J(z, v, u)$ where $z=\Sigma x_{i} y_{i}$.

Case 1. $z=\Sigma x_{i} y_{i}=0$. Then $(u v) \Delta=0$ and since $A=A^{2}$ we have $\Delta=0$.

Case 2. $z=\Sigma x_{i} y_{i} \neq 0$. Then there exists $w \in A$ with $a=z w \neq 0$; otherwise $z F$ would be a nonzero ideal in $A$. Therefore

$$
a \Delta=(z w) \Delta=J(z, w, z)=0
$$

and $K=\operatorname{ker} \Delta \neq 0$. But $K$ is an ideal of $A$. For if $x \in K, y \in A$, then $(y x) \Delta=y \cdot x \Delta=0$ and therefore $K A \subset K$. Thus $K=A$ which means $\Delta=0$. From both of these cases we conclude $C=0$.

Next as for Malcev algebras we have the following

Definition. The set $N=\{n \in A: J(n, A, A)=0\}$ is called the $J$-nucleus of $A$.

Lemma 3.11. If $a, b \in A$ are such that $J(a, b, A)=0$, then $a b \in N$.

Proof. Suppose $J(a, b, u)=0$ for all $u \in A$, then from (3.7)

$$
\begin{aligned}
J(a b, y, z) & =J(y z, a, b)+J(a, b, z) y-J(a, b, y) z \\
& =0 .
\end{aligned}
$$

Corollary 3.12. $N$ is an ideal of $A$ which is a Lie algebra and therefore $N=0$.

Corollary 3.13. $R_{x}$ is a derivation of $A$ if and only if $x \in N=0$.

Now let

$$
D(x, y)=\frac{1}{4}\left[R_{x}, R_{y}\right]-\frac{1}{2} R_{x y}
$$

i.e.

$$
z D(x, y)=[x, y, z]=\frac{1}{2} x y \cdot z+\frac{1}{4}(y z \cdot x+z x \cdot y) \text {. }
$$

Then $D(x, y)$ is an inner derivation, that is, $D(x, y) \in L(A)=\Delta(A, A)$. If $I(A)$ denotes the linear subspace spanned by all such $D(x, y)^{\prime} s$, then we have

Lemma 3.14. The derivation algebra of $A$ equals $I(A)$ and $I(A)$ is completely reducible in $A$. 
Proof. First we shall show that any derivation $D$ of $A$ in $A(A, A)$ is actually in $I(A)$. For let $D=R_{z}+(1 / 4) \Sigma_{i}\left[R_{x_{i}}, R_{y_{i}}\right] \in \Delta(A, A)=L(A)$. Then write $D=R\left(z+(1 / 2) \Sigma_{i} x_{i} y_{i}\right)+\Sigma_{i} D\left(x_{i}, y_{i}\right)$. From this equation $R\left(z+(1 / 2) \Sigma_{i} x_{i} y_{i}\right)$ is a derivation and therefore by Corollary 3.13 it equals zero. Thus $D \in I(A)$.

Next we shall show any derivation of $A$ is in $I(A)$. Since $D$ is a derivation $\left[R_{x}, D\right]=R(x D)$ and therefore using the Jacobi identity we obtain $[\Delta(A, A), D] \subset \Delta(A, A)$. Now the map

$$
\Delta(A, A) \rightarrow \Delta(A, A): X \rightarrow[X, D]
$$

is a derivation of $\Delta(A, A)$. But since $\Delta(A, A)$ is a semi-simple Lie algebra all derivations are inner and therefore there exists $T \in \Delta(A, A)$ so that

$$
[X, D]=[X, T] \text { for all } X \in A(A, A) .
$$

Now since $4(x, y)=\left[R_{x}, R_{y}\right]-R(x y)=4 D(x, y)+R(x y)$ we have, using Corollary 3.13,

$$
\Delta(A, A)=R(A) \oplus I(A)
$$

as a vector space sum. Therefore let $T=R_{z}+D_{1}$ where $D_{1} \in I(A)$ and $z \in A$, then for any $x \in A$,

$$
\begin{aligned}
{\left[R_{x}, R_{z}\right] } & =\left[R_{x}, T-D_{1}\right] \\
& =\left[R_{x}, T\right]-\left[R_{x}, D_{1}\right] \\
& =\left[R_{x}, D-D_{1}\right], \quad \text { using }(3.15) \\
& =R(x \widetilde{D}),
\end{aligned}
$$

where $\widetilde{D}=D-D_{1}$ is a derivation. Therefore

$$
\begin{aligned}
R\left(x\left(\widetilde{D}-2 R_{z}\right)\right) & =R(x \widetilde{D})-2 R(x z) \\
& =\left[R_{x}, R_{z}\right]-2 R(x z) \\
& =4 D(x, z) .
\end{aligned}
$$

Thus $R\left(x\left(\widetilde{D}-2 R_{z}\right)\right)$ is a derivation and using Corollary 3.13 we have

$$
x\left(\widetilde{D}-2 R_{z}\right)=0 \quad \text { for any } x \in A .
$$

However this implies $2 R_{z}=\widetilde{D}$ is a derivation and therefore $0=2 R_{z}=\widetilde{D}$. Thus from the definition of $\widetilde{D}$ we have $D=D_{1} \in I(A)$ so that every derivation of $A$ is in $I(A)$.

The last part of the main theorem is proved in a manner analogous to the proof of Theorem 9 in [1]. We note from (3.16) that the completely reducible Lie algebra $\Delta(A, A)$ is such that the subalgebra $I(A)$ is splittable and has a complementary subspace, namely $R(A)$, 
which is $I(A)$-invariant (because $\left[R_{x}, D\right]=R(x D)$ ). Thus from $[1$, Th. $5], I(A)$ is completely reducible in $A$ and the proof of the main theorem is complete.

\section{REFERENCES}

1. N. Jacobson, Completely reducible Lie algebras of linear transformations, Proc. Amer. Math. Soc. 2 (1951), 105-113.

2. A. Sagle, Malcev algebras, Trans. Amer. Math. Soc. 101 (1961), 426-458.

3. - On derivations of semi-simple Malcev algebras, Portugaliae Math. 21 (1962) 107-109.

4. - Simple Malcev algebras over fields of characteristic zero, Pacific J. Math. 12 (1962), 1057-1078.

5. R. D. Schafer, Inner derivations of nonassociative algebras, Bull. Amer. Math. Soc. 55 (1949), 769-776.

6. K. Yamaguti, On the Lie triple system and its generalization, J. Sci. Hiroshima Univ., Ser. A, 21 (1958), 155-160.

7. - Note on Malcev algebras, Kumamoto J. of Sci., Ser. A, 5 (1962), 203-207.

.University of California, Los ANGeles 



\section{PACIFIC JOURNAL OF MATHEMATICS}

\section{EDITORS}

H. Samelson

Stanford University

Stanford, California

R. M. Blumenthal

University of Washington

Seattle, Washington 98105
J. Dugundu

University of Southern California Los Angeles, California 90007

*Richard Arens

University of California

Los Angeles, California 90024

\section{ASSOCIATE EDITORS}
E. F. BECKENBACH
B. H. NeumanN
F. WOLF
K. YOSIDA

\section{SUPPORTING INSTITUTIONS}

UNIVERSITY OF BRITISH COLUMBIA CALIFORNIA INSTITUTE OF TECHNOLOGY UNIVERSITY OF CALIFORNIA MONTANA STATE UNIVERSITY

UNIVERSITY OF NEVADA

NEW MEXICO STATE UNIVERSITY

OREGON STATE UNIVERSITY

UNIVERSITY OF OREGON

OSAKA UNIVERSITY

UNIVERSITY OF SOUTHERN CALIFORNIA
STANFORD UNIVERSITY

UNIVERSITY OF TOKYO

UNIVERSITY OF UTAH

WASHINGTON STATE UNIVERSITY

UNIVERSITY OF WASHINGTON

AMERICAN MATHEMATICAL SOCIETY CALIFORNIA RESEARCH CORPORATION SPACE TECHNOLOGY LABORATORIES NAVAL ORDNANCE TEST STATION 


\section{Pacific Journal of Mathematics}

\section{Vol. 15, No. $1 \quad$ September, 1965}

Donald Charles Benson, Unimodular solutions of infinite systems of linear

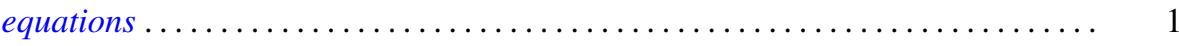

Richard Earl Block, Transitive groups of collineations on certain designs . . . . . . 13

Barry William Boehm, Existence of best rational Tchebycheff approximations .... . 19

Joseph Patrick Brannen, A note on Hausdorff's summation methods . . . . . . . . . . 29

Dennison Robert Brown, Topological semilattices on the two-cell ............ 35

Peter Southcott Bullen, Some inequalities for symmetric means . . . . . . . . . . 47

David Geoffrey Cantor, On arithmetic properties of coefficients of rational

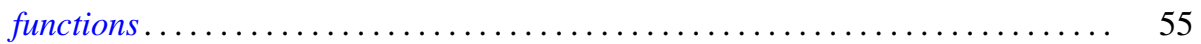

Luther Elic Claborn, Dedekind domains and rings of quotients . . . . . . . . . 59

Allan Clark, Homotopy commutativity and the Moore spectral sequence ........ 65

Allen Devinatz, The asymptotic nature of the solutions of certain linear systems of

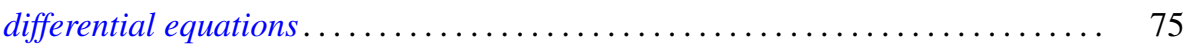

Robert E. Edwards, Approximation by convolutions ................... 85

Theodore William Gamelin, Decomposition theorems for Fredholm operators . . . . . 97

Edmond E. Granirer, On the invariant mean on topological semigroups and on

topological groups .................................. 107

Noel Justin Hicks, Closed vector fields . . . . . . . . . . . . . . . . . . . 141

Charles Ray Hobby and Ronald Pyke, Doubly stochastic operators obtained from

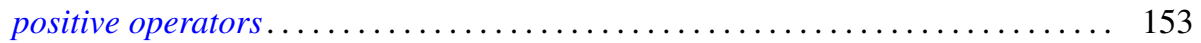

Robert Franklin Jolly, Concerning periodic subadditive functions . . . . . . . . . 159

Tosio Kato, Wave operators and unitary equivalence . . . . . . . . . . . . . . 171

Paul Katz and Ernst Gabor Straus, Infinite sums in algebraic structures . . . . . . . 181

Herbert Frederick Kreimer, Jr., On an extension of the Picard-Vessiot theory ...... 191

Radha Govinda Laha and Eugene Lukacs, On a linear form whose distribution is

identical with that of a monomial ......................... 207

Donald A. Ludwig, Singularities of superpositions of distributions . . . . . . . . . 215

Albert W. Marshall and Ingram Olkin, Norms and inequalities for condition



Horace Yomishi Mochizuki, Finitistic global dimension for rings . . . . . . . . . . 249

Robert Harvey Oehmke and Reuben Sandler, The collineation groups of division

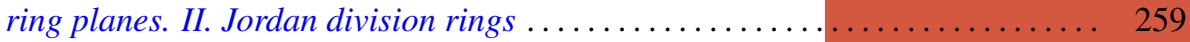

George H. Orland, On non-convex polyhedral surfaces in $E^{3} \ldots \ldots \ldots \ldots \ldots \ldots \ldots 267$

Theodore G. Ostrom, Collineation groups of semi-translation planes . . . . . . . . 273

Arthur Argyle Sagle, On anti-commutative algebras and general Lie triple

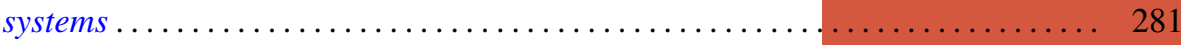

Laurent Siebenmann, A characterization of free projective planes . . . . . . . . . 293

Edward Silverman, Simple areas.................................. 299

James McLean Sloss, Chebyshev approximation to zero .................. 305

Robert S. Strichartz, Isometric isomorphisms of measure algebras . . . . . . . . . 315

Richard Joseph Turyn, Character sums and difference sets . . . . . . . . . . . . 319

L. E. Ward, Concerning Koch's theorem on the existence of arcs . . . . . . . . . . 347

Israel Zuckerman, A new measure of a partial differential field extension ......... 357 